\title{
6.3B Scientific Background of Physical and Rehabilitation Medicine: Specificity of a Clinical Science
}

\author{
Luigi Tesio ${ }^{1,2}$ \\ 'Department of Biomedical Sciences for Health, Physical and Rehabilitation Medicine, The University of Milan, Italy, ${ }^{2}$ Istituto Auxologico Italiano, IRCCS, \\ Department of Neurorehabilitation Sciences, 20122 Milano, Italy
}

\section{INTRODUCTION}

The dominant scientific model of medicine is biomedical. Medicine is seen more and more as biology applied to man. Biology, in turn, is more and more indebted to chemistry and physics.

In the end, physics has become the hallmark and the main "paradigm" (as per Thomas Kuhn's successful terminology ${ }^{[1]}$ ) of what is meant by "science." In philosophical language, this paradigm can be simplified to two terms: reductionism and determinism.

Reductionism indicates that parts underlie the whole. Reductionism can be seen under two related, yet distinct, perspectives. From a backward perspective, multiple phenomena (e.g., symptoms) can be "reduced" to a common cause (e.g., a given disease). From a forward perspective, any single unitary phenomenon ("what is appearing," according to the Greek etymology) can be split into components. The interactions between these components, organized hierarchically along levels of complexity, realize a "mechanism." ${ }^{.2]}$ In the medical language, the backward reasoning can be translated into the search for "aetiology" (the ultimate or, at least, a "deep" cause, the "what"); the forward reasoning can be translated into the concept of "pathogenesis" (the "how").

Determinism assumes that universal laws bind the parts and the whole and make their interactions fully predictable. Discovering the eternal universal behind the transient particular is the ultimate goal of the scientific enterprise. Realizing observations after removing interferences or confounding factors ("diffalcare gli impedimenti" as per the Galilei's language, ${ }^{[3]}$ ) became the ultimate goal of modern and contemporary experiments. By the way, both observational-epidemiological and manipulative-experimental methods, including "thought" experiments, ${ }^{[4]} \mathrm{imply}$ procedures minimizing Galilei's "impediments," in the endeavor to support the cause-effect inference.

\begin{tabular}{|l|l|}
\hline \multicolumn{3}{|c|}{ Access this article online } \\
\hline Quick Response Code: & Website: \\
\hline & www.jisprm.org \\
\hline
\end{tabular}

This model, born in the $17^{\text {th }}$ Century, was extremely successful in all sciences. In medicine, it provided a sudden advancement in knowledge and, in the $20^{\text {th }}$ century, an astonishing advancement in treatment efficacy. Many of the readers of this chapter probably are still alive, just thanks to the successes of this model. From 1901, the list of Nobel prizes for "Physiology or Medicine" testifies that the model works; it cannot be renounced; it by far the best we, humans, have.

\section{Physical Medicine and Rehabilitation: The Cinderella of Biomedical Sisters}

Having said that, a critical look at the medical variant of the reductionist-determinist model may explain why physical and rehabilitation medicine (PRM) together with a handful of other specialties, still suffers from a "Cinderella" syndrome. ${ }^{[5]}$ The syndrome includes a low "impact" of its publications across the scientific community ${ }^{[6]}$ (whatever metrics is adopted); the low "prestige" assigned to the specialty by both lay and professional people; $;^{[7,8]}$ and the relatively scarce support to research by public and private funding agencies. The syndrome includes a loss of self-esteem in PRM practitioners and researchers. ${ }^{[9]}$ The privileged Cinderella's sisters are the "bio"medical specialties, such as Neurology, Cardiology, and Oncology. The syndrome is revealed by the Greek origin of the name itself of the affected specialties. The "-logy" specialties are exempt, the "-iatry" specialties are affected. The "-logy" suffix comes from "lògos," indicating "language, word" in the high sense of "giving name, hence meaning" to reality. It indicates understanding and rational knowledge of reality and ultimately of truth. The name intelligence has the same root. In medicine

Address for correspondence: Prof. Luigi Tesio, Istituto Auxologico Italiano, Riabilitazione Neuromotoria, via Giuseppe Mercalli, 30, 20122 Milano, Italy. E-mail: luigi.tesio@unimi.it

This is an open access journal, and articles are distributed under the terms of the Creative Commons Attribution-NonCommercial-ShareAlike 4.0 License, which allows others to remix, tweak, and build upon the work non-commercially, as long as appropriate credit is given and the new creations are licensed under the identical terms.

For reprints contact: reprints@medknow.com

How to cite this article: Tesio L. 6.3B Scientific background of physical and rehabilitation medicine: Specificity of a clinical science. J Int Soc Phys Rehabil Med 2019;2:S113-21. 
an extremist interpretation of the reductionist-determinist model ascribes "lògos" to specialties dealing with body parts and the predictable laws governing their interactions. "Truth" is behind the human phenomenon, hidden by human behaviors and perceptions. Surgical specialties are biased by a more empirical approach: "sur" comes from "chèir," meaning hand; "gery" comes from "ergon," meaning making or repairing. Yet, their reductionism (surgery has to do with body parts, after all) make these specialties acceptable within the drawing room of the lògos family. The tiniest the body parts and the more general and ubiquitous their role in biology (reductionism), and the stronger their binding laws (determinism), the more "scientific" the corresponding specialties are considered: hence it comes the growing status of specialties focused on cells (oncology) or even molecules (immunology, genetics). These "molecular" specialties are progressively overshadowing the less reductionist "organ" specialties. This heralds the worrying decadence of all "clinical" sciences, i.e., those requiring a one-to-one, patient-physician personal relationship, again as per the Greek etymology. "Klìnein" - same root as inclination and decline-means lying as well as leaning (toward a bedridden patient, of course $)^{[6,10]}$ Here comes also the progressive fractioning and loss of clinical attitude of organ specialties: for instance, interventionist cardiology, on one side, and cardiac electrophysiology and arrhythmology, on the other side, are becoming sub- (or super-?) specialties gradually eroding cardiology.

\section{"Iatric" Specialties: Are they Specialties?}

The "iatry" suffix, coming for the Greek "iatròs" (simply: the one who is curing), does not specify any target organs (to say nothing of underlying cells or molecules). The iatric specialties deal with the person as a whole. This is the case for PRM ("Physiatry"), psychiatry, geriatrics, pediatrics, phoniatrics, and for a handful of other specialties with a different name but a similarly ill-delimited mission, like for example sports medicine, and occupational and industrial medicine. This makes these specialties to appear generic and "symptomatic." They seem related to caring, not curing. Someone may claim that they are "more than science," given that they are "holistic," require skilled intuition, and are endowed with a high philanthropic mission: unfortunately, "more than," barely conceals here an "other than" meaning. A closer look shows that most of these iatric specialties are not entirely generic. For all but PRM, a delimited focus can be easily detected: for example, mind for pyschiatry, age for geriatrics and pediatrics. Within this wide, yet delimited perspective, a reductionist approach can be rescued at least partially: for instance, by emphasizing research on brain imaging and neurophysiology, for psychiatry, and cell-molecular research on age-specific diseases, for geriatrics and pediatrics. The paradox of a generic specialty, however, remains untouched for "physiatry," still claiming to span across mind and body, all age classes, and all kinds of human activities (from daily living to work and sports) [See Appendix 1 for examples of PRM definitions internationally].

\section{Physical and Rehabilitation Medicine: Time for Saying What It Is Not, Not Only What It Is}

Before highlighting the scientific foundations of the "unspecific specialty" of PRM one must admit that considerable efforts have been made to define it. Perhaps in the endeavor to assert its challenged scientific dignity, all definitions on one hand claim for the widest possible boundaries, spanning across all ages and pathologic conditions while, on the other hand, intentionally leave these wide boundaries rather blurred. Appendix 1 reports six examples from which it is evident that the (correct) claim for a wide scope is biased by vagueness in defining boundaries. To cite the most important case, blurred boundaries appear those between the medical and the social competences to be involved in "rehabilitation," and between the missions of research on diseases, versus research on their functional consequences (i.e., impairments, activity limitations, and participation restrictions, as per the 2001 World Health Organization ICF glossary).${ }^{[1]}$ Unfortunately, blurred boundaries pave the way to confusion. Sharp boundaries across concepts would rather be the requisite for their integration.

The key to overcome ambiguities is acknowledging that defining its specificity is a priority for PRM. What PRM is cannot be convincingly asserted without asserting what PRM is not.

\section{The Words Count: Physical Medicine Declares the Means, Rehabilitation Declares the Goals}

A first reflection relates to the name itself of the specialty. It has been convincingly sustained that "physical" must be interpreted in the original and wide Greek sense. ${ }^{[10]}$ The Greek name "fissis" mostly means nature, in the sense of "outer, natural world," although it may also have the meaning of "intimate essence of" (a meaning overlooked here). "Physical" therapies thus are those administered "from outside, onto the person as a whole." Muscular electrostimulation, gait training, and speech therapy are thus all equally "physical." Physical medicine might be said to be a form of "external" medicine. Its counterparts are not "chemical medicines" (a nonexisting term) but, rather, all forms of "internal" medicine (from drug treatments to surgery). Rehabilitation means providing the disabled person with "ability," the best possible interaction with the outer environment (inclusive of other persons).$^{[1]}$ In a sentence, physical medicine declares the means, rehabilitation declares the goals.

Based on this statement a further attempt has been made in the form of a provoking "specificity game,"[12] summarized here by Figure 1 and Table 1. This asks for yes/no decisions, and thus it is fully qualitative, reminiscent of Aristotelic logic, perhaps filtered by a scholastic and late renaissance elaboration, ${ }^{[13]}$ yet in any case conceived uphill the "quantitative" revolution triggered by Galileo, Décartes and Newton. This choice was intentional. Let us admit that at this stage, PRM needs more definitions than equations, more logic than calculus. 
In Figure 1 continuous $\mathrm{X}-\mathrm{Y}$ axes are proposed yet, in essence the game is qualitative. Along the $\mathrm{X}$ axis a gradient of "rehabilitativeness" is represented: the more the goal is restoring (in the case for therapeutic procedures) or measuring (in the case for diagnostic procedures) a successful person-world interaction, the more rightward should the procedure be placed. The Y axis represents a gradient of "physicality" of the procedure: the more it acts from outside on the whole person (therapies) or the more it captures person-environment relationships rather than within-body events (diagnostics), the higher should the procedure be placed. For simplicity, the X-Y frame is divided into four quadrants with equal areas. Obviously, the "specific" PRM procedures are those placed into the upper-right, gray-shaded quadrant (high "physicality" of means combined with high "rehabilitativeness" of goals). "Guessing the quadrants" is the target of the game (here comes its qualitative nature). In this Figure 1 motor exercise and language testing (a therapeutic and a diagnostic procedure) are both placed in the "specific" PRM quadrant. These choices should be easy to be agreed upon. Both of the procedures aim at rehabilitation and, at the same time, both are "physical" (although "language" is conventionally ascribed to the "cognitive," as distinct to the "motor," domain) in the wide sense of acting from the outer world on the person as a whole. In the original published game questions related to 10 other therapeutic and 10 other diagnostic procedures were asked $^{[12]}[$ Table 1].

Of course, in case the questioned healthcare professionals did not know the procedures listed, these had to be explained in advance. For each question, the "correct" quadrant placement was also suggested, simply based on the opinion of the author. The curious reader can easily play the game and/ or administer the test to other professionals. He/she may also raise different questions. The proponent will invariably observe that, whichever the questions and, as a rule, their preset "correct" answers, "iatric" specialists tend to get higher scores, compared to "logist" specialists. It is surprising to see how PRM professionals find difficulties reaching a substantive agreement. The most controversial points usually concern procedures which appear "specific" according to one axis but not according to the other (unpublished observations). For instance, the injection of Botulinum toxin against spasticity is widely adopted in PRM, where it has rehabilitation goals: yet, it is difficult to detect that it is a form of "internal" medicine (on the other hand, that is precisely why it is widely practiced in many other specialties, from neurology to ophthalmology). This is not to say that PRM specialist should not inhabit, in their practice, the other quadrants. They are physicians and as such, they are fully entitled to exploit all of the resources offered by the biomedical sciences. The open challenge, however, remains one of delimiting the core competences making PRM unique.

This game highlights that finding consensus on what it is and what it is not specific to PRM still is a necessary step to provide the specialty and its professionals with a stronger identity. Only after

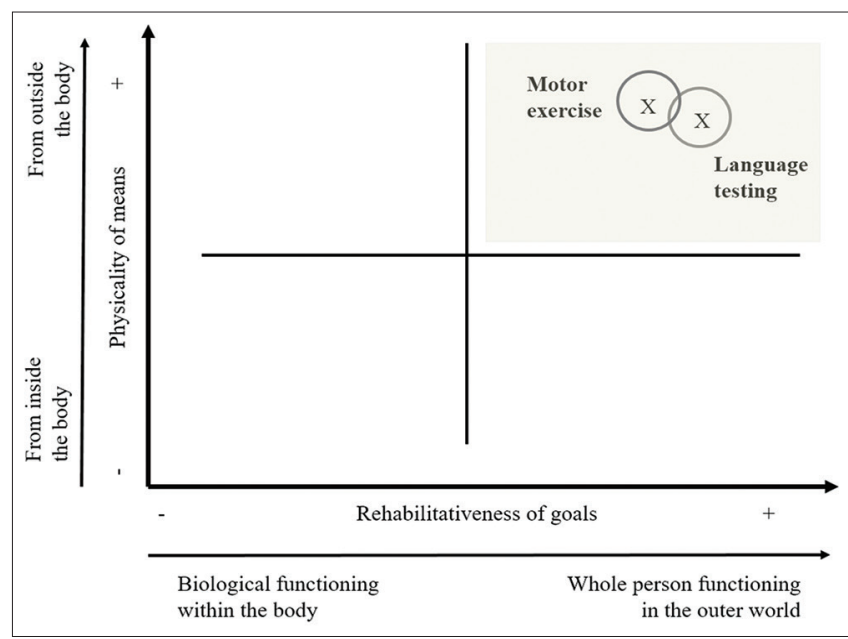

Figure 1: The "physical and rehabilitation medicine specificity game." Therapeutic and diagnostic procedures can be plot on a X-Y frame. The $X$ axis represents a gradient of "rehabilitativeness" of the goals, i.e., how much they aim at improving or diagnosing person-environment interaction (rightmost positions), rather than within-body organ interactions (leftmost positions). The $Y$ axis represents a gradient of "physicality" of the means, i.e., how much do they act from outside the body on the person as a whole rather than from within the body, from top to bottom, respectively. For simplicity, the plane is divided into 4 quadrants with the same area. For any given procedure the game consists in guessing the "right" quadrant (preset by the game proponent). The upper right, gray-shaded quadrant encases the procedures most specific for physical and rehabilitation medicine: as clear-cut examples, motor exercise and language testing are both placed here ${ }^{[12]}$ (modified) Table 1: A list of therapeutic and diagnostic procedures.
Are they specific to PRM?

\begin{tabular}{ll}
\hline Therapeutic procedure chart & Diagnostic procedure chart \\
\hline Antidepressant drugs & $\begin{array}{l}\text { Basal metabolic rate } \\
\text { measurement }\end{array}$ \\
Antihypertensive drugs & Bicycle stress ECG \\
Botulinum toxin injection for spasticity & Brain fMRI \\
or dystonia & \\
Muscle strengthening exercise & Gait analysis \\
Laser corneal ablation for myopia & Handgrip force testing \\
Shock waves for delayed union of & Needle electromyography \\
fractures & \\
Speech therapy & Spatial neglect testing \\
t-DCS & Surface dynamic \\
& electromyography \\
Tendon transfer in tetraplegia & Urodynamics \\
PTBS for bladder incontinence & Videofluoroscopy for \\
& swallowing \\
\hline
\end{tabular}

The 10 therapeutic and the 10 diagnostic procedures proposed in the original "PRM specificity game,"[13] recalled by Figure 1. The original article also proposed the "right" answers and the cut-off cumulative scores expected from different kinds of specialists. The interested reader can try to place the procedures into the "correct" quadrant suggested by the Author in the original article. t-DCS: Transcranic direct-current brain stimulation, ECG: Electrocardiography, fMRI: Functional MRI, MRI: Magnetic resonance imaging, PTBS: Percutaneous tibial nerve (electrical) stimulation

the identity has been sharply defined its scientific foundations can be reliably established and then loudly claimed for. 
The Scientific Foundations of the Biomedical versus the Clinical-Behavioral (InCluding Physical and Rehabilitation Medicine) Model

Two corollaries of the strict biomedical scientific model must be highlighted.

a. Individuals (be they atoms, molecules, cells, organs, persons) have no real "initiative" (as per the so-called "universal determinism" of Laplace). They respond predictably to causal stimuli. The actual capacity for predictions of specific events is an empirical, not a theoretical, issue

b. Different individuals across a population are equivalent. A given protein, a given blood cell, a given axon or slice of liver, and a given inbred rat can be considered equivalent to other specimens of the same kind, and adequately represented by their (virtual) corresponding "mean" individual, except for random variation.

By contrast, it is quite clear that PRM, having a clinical mission of individual treatment, must accept the single person, empirically appearing as pro-active, indivisible, and unique, as its unit of observation and treatment [hence the specificity of the upper right quadrant of Figure 1]. This implies that whole person's behaviors and perceptions must be set as the specific goals of research and treatments. For instance, consider the procedures that might be placed in the two lower quadrants of Figure 1. "Internal" and/or local treatments frequent in the PRM practice (such as drug administration and muscle stretching for spasticity) aim nonetheless at whole-person outcomes (e.g., better mobility, decreased pain), not at local outputs. The same holds for the upper left quadrant of Figure 1, reserved to "physical" procedures, yet devoid of rehabilitation goals so that it cannot be "inhabited" by physiatric procedures. Take the examples of acoustic shock-waves against kidney stones and dynamic calcaneus traction applied to a fractured leg. The person is, of course, also a replicable biological entity yet, if one wants to cure that particular individual, he/she must be credited with some initiative, making him/her rather unpredictable and unique: in other words, he/she behaves as a subject. How much unique can be the human beings? Don't they share 99\% of their genome with Apes, after all? Of course, persons are more distant, both in biology and behaviors, from molecules and cells than from Apes. The distance is huge even from their closest evolutionary neighbors, however. The concept of distance is cultural not less than biological. The largest, perhaps unfillable, gap is marked by the man-specific behavioral and symbol-based (in a word: cultural) "dimension" of evolution. These for sure enrich the too rigid Darwinian model. ${ }^{[13]}$ In retroaction the cultural evolution itself has biological effects. The diseases affecting the human kind not only determine but, in a closed circuit, are also determined by the evolving cultural and social context. Anthropological studies convincingly demonstrated that this was true in the early millennia of human migration from eastern Africa to the rest of the world, ${ }^{[14]}$ at the recent dawn of the western civilization, ${ }^{[15]}$ and it is still true in our declining industrial era. Even more: however counterintuitive it may appear, the definition itself of what is a disease worth of prevention and treatment, rather than a "normal" and acceptable variant of the human condition, is highly culture-dependent. ${ }^{[16]}$ Cultural evolution not only introduces a gap within the biological evolution but, also, exacerbates the individual peculiarities within the human kind. Be these peculiarities "determined" or spontaneous, no clinical specialty can mistake population or mean virtual individuals for concrete single individuals.

The problem of defining the scientific foundations of PRM medicine thus simplifies to the problem of defining (a) which of the methods of biomedical research should be given priority when imported into a human behavioral science and (b) which methods should be imported from other cultural domains. The latter point is the most critical, of course, and will be given special evidence heretofore.

\section{Physical and Rehabilitation Medicine Research: Distinct, Not Alternative}

The three prioritary themes of scientific research requiring a specific approach are (a) the variables to be defined and observed; (b) statistics; and (c) the trial designs. Actually, some of these are simply variants of those encountered in biomedical sciences, while some are specific of the so-called "human" sciences (yet: sciences, nonetheless).

Table 2 summarizes the specificity of the PRM model, compared to the biomedical model.

\section{Selecting the "variables of the person"}

This is often the starting point in PRM research. Behavioral variables cannot be substituted for by any "surrogate" physical parameter. Walking speed cannot replace independence in walking and in daily life. Brain sensory evoked potentials cannot replace a subjective report of pain. Similar reasoning applies to fatigue, balance, continence, depression, communication skills, and the like. Nevertheless, these variables are looked at very suspiciously by the biomedical scientists who suspect that they lack the very requisite of existence (if scientists adhere to an extreme empiricism), or at least of inter-rater reliability ("objectivity") if scientists adhere to a more moderate empiricist-realist view according to which if these variable exist, they cannot be a source of scientific knowledge, anyway (for a deep philosophical reflection on "objectivity" see $\left.{ }^{[17]}\right)$. The error lies in thinking that the risk for being an artificial mental construct only affects "person-behavioral-psychic" variables, not physical variables. First, the bio-medical extremists mistake a matter of historical precedence for one of conceptual priority. The history of variables such as force, temperature and energy was highly troubled, not less than that of, say, intelligence and depression, ${ }^{[18]}$ and many errors and ambiguities had to be overcome along the past centuries. Second, they overlook the fact that knowledge in itself is a mental process. Few would deny that "out there" objects exist and interact quite independently of thinking humans. The problem is that 
everything interacts with everything. Yet, "giving the name" to objects and discovering specific relationships among objects (which humans presumptuously call "laws") through tiring and skillful experiments allowing to remove Galilei's impediments is always a mental process. This philosophical position, called by someone realism-perspectivalism, ${ }^{[19]}$ is essentially a realistic view of the world, yet mitigated by an empiricist approach depriving man from his omnipotence but leaving him with an intact responsibility on its interaction with the world, including its unique capacity for manipulation based on scientific knowledge. If this reasonable standpoint is accepted the inferior status of person variables vanishes, thus paving the way for growing financial investments in PRM and, most important, for further cultural reflections. At last, the proper relevance will be given to topics such as, for instance, the development of appropriate outcome measures, and the refinement of the recent theory (imbued with statistical models) of "latent" person's variables, including the dilemma on which latent variable is "real" and which is purely a mental artifact. ${ }^{[20,21]}$

\section{Statistics}

This, too, has long been looked at suspiciously by bio-medical enthusiasts. Claude Bernard, the great physiologist who founded the contemporary "experimental medicine," considered it as a form of cosmetic surgery striving to accommodate data coming from a wrong hypothesis or a failed experiment, when they did not fit a deterministic law. ${ }^{[2]}$ Nowadays, perhaps with the exception of the most orthodox "bernardian" field of physiology, an ingenuous opposite attitude emerged in biomedicine. Statistical "significance" (a given difference between observations being assumed as nonchance) is often mistaken as the highest form of "evidence" for a cause-effect relationship. ${ }^{[23]}$ Another common error is the simplistic manipulation of ordinal scores from questionnaires ${ }^{[24]}$ (including the popular one-item questionnaires often trivialized as "visual analog" segments ${ }^{[25]}$ ). These are counts of events, the homogeneity and the actual "weight" of which remains to be determined. Beyond its misuse when applied to populations in behavioral research, another key error about biostatistics is a simplistic application to single persons. Biostatistics is focused on populations. It assumes that central indexes, albeit surrounded by random error (usually the smaller, the greater the sample), can validly represent the ensemble of (replicable) individuals within the population. By the way, replicable individuals cannot be limited to molecules or cells. These can also be human beings, if research is primarily aimed at populations: this is the case for the areas of public health, epidemiology and preventive medicine which, not surprisingly, share with biomedicine most of the measurement paradigms and a higher scientific consideration, compared to $\mathrm{PRM}^{[6]}$ [Table 2]. PRM, like any clinical activity, has to do with human individuals, much less predictable than population means. Therefore, its development lies in the priority assigned to two statistical topics:

a. Estimating the metric validity (tested in terms of unidimensionality, reliability, accuracy and precision) of measures of person's variables and b. Estimating how reliably can measures of change coming from cohort studies be applied to single individuals.

Solutions are available. Person's variables are usually (and often unavoidably) measured through cumulative questionnaires, providing ordinal scores with very limited metric validity. Yet, the new emerging item-response statistical theory (in particular the growing Rasch analysis) may offer a solution of unprecedented validity and practical usefulness. ${ }^{[26]}$ The reliability of individual changes can be estimated from prior independent cohort studies, if proper indexes such as the "minimal real difference" are computed. ${ }^{[27]}$ Counting the improved individuals, rather than computing the "mean" improvement across people (some of which probably worsened, and not by chance!), may provide a wider, and often more sensible, perspective to PRM studies.

\section{Trial design}

This topic is often underestimated and confused with respect to statistics in its proper sense. It is the set of procedures (the various forms of "control") aimed at minimizing the interference of unknown (random and/or systematic) causes on the outcome of an observational or an experimental study. For instance, blindness to the treatment arm (i.e., the one receiving "true" vs. placebo drug) aims at minimizing selection biases, rater's prejudice, etc. The randomized, controlled trial (RCT) design, mostly in its "double blind" version, applied to groups of individuals, correctly emerged as the gold standard in biomedical research. Unfortunately it is not applicable to many types of PRM studies: for example, where blindness is impossible like in testing manual treatments or where the nonrandom selection of a specific therapist, due to empathy of competence considerations, is an ingredient of the therapy or, where therapy is made of a mix of ingredients selected through a personalized decision-tree procedure, ${ }^{[28]}$ etc. An overoptimistic consideration of the RCT has been often criticized ${ }^{[29]}$ sometimes with elegant irony. ${ }^{[30]} \mathrm{A}$ vast and sophisticate array of so-called "quasi experimental" designs, coming from the world of psychological and social sciences, can be easily imported into PRM..$^{[31]}$ Efforts should be made to implement and exploit these designs, the validity of which is often underestimated in favor of the more popular, yet often less appropriate, biomedical designs.

\section{What Physical and Rehabilitation Medicine Is Nот}

It is now time to claim open for what PRM is not, without fear for being denied any identity. Only three prioritary claims (concerning what PRM is not) will be highlighted here.

\section{Physical and rehabilitation medicine is not "holistic:" Core} competences are motor and functional

PRM is a bilingual specialty. It has its roots in bio-medicine, its branches in behavioral sciences exploring human activities and perceptions. This is both its charm and its curse. Its deepest conceptual roots are in physiology and more precisely, in the 


\begin{tabular}{ll}
\hline $\begin{array}{l}\text { Qriables } \\
\text { Question: What do I }\end{array}$ & $\begin{array}{l}\text { Clinical medicine and PRM } \\
\text { want to observe? }\end{array}$ \\
$\begin{array}{l}\text { E.g., independence, fatigue, pain, balance, } \\
\text { communication etc., often recorded as } \\
\text { items in questionnaires }\end{array}$ \\
\\
\\
"Latent": Their quantity can only be \\
inferred from counts of a sample of \\
behaviors representative of the individual's \\
property. E.g., counts of responses to a \\
questionnaire may indicate more or less \\
pain, or independence in daily life etc. \\
Ordinal, discrete. Each response may be \\
counted as "one more", yet its individual \\
"weight" is unknown. Not only errors \\
in the observation, but also inferences in \\
the transformation of raw scores (which \\
are sum of counts of dichotomous \\
observations) in measurements as proxies \\
of the true hidden "quantity"
\end{tabular}

Table 2: Variables, statistics and trial designs a logical gradient from body parts to persons' populations

Statistics

Question: Could what I observed happen by chance alone?

Trial design

Question: What I observed did not happened by chance. But: Did it happen because of an unsuspected cause? (so called "third variable explanation")

Bio-medicine

Objective, observed in individuals. E.g., blood pressure, glycemia, and nerve conduction velocity.

"Manifest": Entirely open to observation. Their individual quantity is directly measurable

Most are linear, continuous. High precision and reliability through instrumental measurement, small error in the observation

\begin{abstract}
Transformation from sum of counts to estimate linear measurement required. E.g., Rasch analysis ${ }^{[2]}$
\end{abstract}

Uniqueness of the person. Averaging criticizable, hence error in individual measurement not attenuated

Adjustments and standardizations criticizable. Individual peculiarities are substantive (e.g., in deciding treatment). Outcomes are often discontinuous events (e.g., return to work, discharge home etc.). Logistic regression and interaction-based modeling are more appropriate (e.g., neural networks, classification and regression trees)

Effect sizes moderate, sample sizes small, side effects moderate. Statistics should highlight power (enhancing the true positive risk) not less than significance (abating the false positive risk)

Patient-therapist interaction is part of the treatment, not a source of error variance

Single and standard treatments rarely applicable. Individual treatment comes from decision-tree prescription algorithms Blindness and placebo rarely applicable Individual ethics prevailing
Measurement units valid in themselves. E.g., length, weight, voltage, and time

Averaging can smooth measurement error. Inferences can be modeled (e.g., Gaussian distribution, confidence limits etc.)

Adjustments and standardization across individuals may foster the detection of the effectiveness of an intervention on individuals. Conventional variance/ covariance statistics and multiple regression modeling are appropriate

Effect sizes highlighted by reduction of error variance. Side effects potentially dangerous. Statistics is privileging significance (abating the false-positive risk)

Blindness, group assignment, randomization, stratification and standardization of treatment can often counteract most sources of bias

Public health

Latent or manifest variables, estimated in samples and populations, not in individual subjects. E.g., mean survival time, mean independence in a class of patients, mean risk of developing a disease
Same as for biomedicine, applied to samples and populations (except for rare epidemiologic studies on latent variables)
Same as for biomedicine, applied to samples and populations in prospective studies. Less control in retrospective, case-control, and cross-sectional studies

Individual and population ethics equivalent Population ethics prevailing Summary of the analogies and the differences between the scientific paradigms of physical and rehabilitation medicine, bio-medicine, and public health sciences, from left to right, respectively. It is evident that biomedicine and public health share most of their research paradigms, given that they are not primarily aimed at the single, concrete individual (be it a molecule, a cell or a citizen) but to populations or, quite equivalently, the virtual "mean" individual. The comparison across columns should highlight that specificity of PRM does not place it outside the main stream of contemporary research. Simply, its paradigms are not confined to the biomedical variant and its close sibling, the public health variant, of the more general scientific model ${ }^{[6,10]}$ (modified) 
physiology of the nervous system and of the musckuloskeletal apparatus, i.e., the structures allowing the interaction of the individual with the outer environment. These roots are not as deep in other organ/disease-bound specialties such as Neurology or Orthopedics (which is not to deny that individual Physiatrists may come from the most various curricula). Physiology is a strange, charming nonspecialty, which can be said of "how" it works, but not precisely "on what" it works. PRM inherited from physiology the "how" (i.e., a sound experimental attitude) yet it exploited its own "what" in the difficulties of the person-world interaction. Therefore motor impairments (inclusive of the underlying neurologic and muscle-skeletal disorders) provide its core targets for basic and clinical research. Within the person-world interaction, however, movement is just the mean to realize various goals (in themselves, mental-social constructs), including communication with other persons. There are no internal states that can be communicated without an interpretable motor behavior (be it speech or gesture), perhaps with the exceptions of communication of motor commands though brain-computer interfaces ${ }^{[32]}$ or communication of "consciousness" through brain-MRI interfaces ${ }^{[33]}$ (a much more controversial issue, however, revolving around the definition of consciousness). ${ }^{[34]}$ For sure, all internal states can influence the motor behavior. Communication is crucial to both the diagnosis of motor impairments and their treatments, in particular if consisting in a teaching/learning relationship. For all of these reasons, PRM cannot renounce to explore "nonmotor" domains (actually, becoming manifest through motor behaviors) such as those of pain, cognition, psychology and psychiatry: in so doing PRM should always look at their specific relevance with respect to the person-world interaction.

\section{Physical and rehabilitation medicine is not a sub-specialty} of organ-based specialties

"Cardiac," "Pulmonary," "Oncologic" rehabilitation and the like are misleading terms. Physiatrists must take into account, of course, organ diseases, in order to achieve their primary rehabilitative goals. However, this is different from being specialists in those organ-based diseases. On the other side of the same coin other specialists may well add some "physical therapies" to their therapeutic armamentarium (e.g., aerobic exercise for heart-infarcted patients); however, this does not transform cardiology into PRM. Of course, there may be cases where equal levels of both organ-oriented and PRM competences are required: there are no reasons why collaboration in these cases should imply confusion.

\section{Physical and rehabilitation medicine is neither an alternative nor a "complementary" medicine}

PRM shares with "complementary medicines" some reluctance to split the motor, the cognitive, the psychologic and the social aspects of illness [Table 2 for a clearer conceptualization of this point]. However, by no means it shares the substantial rebuttal of the experimental method. ${ }^{[35]}$ Actually, this method might be applicable to many forms of complementary medicine, ${ }^{[36]}$ although in the wider sense described in Table 2. Unfortunately this seems not a priority for their supporters. In any case, "alternatives" to the contemporary scientific model are not justified.

\section{Conclusion}

The scientific foundations of PRM medicine are the same of contemporary bio-medicine, rooted in the experimental method born in the $17^{\text {th }}$ century. In the meanwhile the PRM specific focus on the person-world interaction requires adaptation of the biomedical methods to face the uniqueness of each person, his/her unpredictability due to his/her capacity to generate behaviors on the basis of internal psychic states, and the need to take into account his/her social needs and subjective value judgments. This requires an open mind. Physiatrists should accept to import into their medical specialty the vast scientific knowledge accumulated in psychological and social sciences. This is possible and has nothing to do with alternative medicine: it simply represents the correct application, and the deepening and widening, of the foundations of contemporary science.

\section{Financial support and sponsorship}

Nil.

\section{Conflicts of interest}

There are no conflicts of interest.

\section{References}

1. Kuhn TS. The Structure of Scientific Revolutions. Chicago, IL: University of Chicago Press; 1962.

2. Craver CF. Functions and mechanisms: A perspectivalist view. In: Huneman P, editor. Functions: Selection and Mechanisms. Berlin: Springer; 2013. p. 133-58.

3. Galilei G. Dialogues on two chief world systems-Ptolemaic and Copernican. 1632, in Italian. English Translation by Drake S., with a foreword by Albert Einstein. Berkeley and Los Angeles, CA. University of California Press; 1967.

4. Buzzoni M. Thought experiments from a Kantian point of view. In: Brown JR, Frappier M, Meynell L, editors. Thought Experiments in Philosophy, Science, and the Arts. Vol. 1. London/New York: Routledge; 2013. p. 90-106.

5. Tesio L, Gamba C, Capelli A, Franchignoni FP. Rehabilitation: The Cinderella of neurological research? A bibliometric study. Ital J Neurol Sci 1995;16:473-7.

6. Tesio L. The good-hearted and the clever: Clinical medicine at the bottom of the barrel of science. J Med Pers 2010;8:103-11.

7. Album D, Westin S. Do diseases have a prestige hierarchy? A survey among physicians and medical students. Soc Sci Med 2008;66:182-8

8. Creed PA, Searle J, Rogers ME. Medical specialty prestige and lifestyle preferences for medical students. Soc Sci Med 2010;71:1084-8.

9. Cole TR, Carlin N. The suffering of physicians. Lancet 2009;374:1414-5.

10. Tesio L, Franchignoni F. Don't touch the physical in "physical and rehabilitation medicine". J Rehabil Med 2007;39:662-3.

11. World Health Organization. International Classification of Functioning, Disability and Health. Geneva: World Health Organization; 2001.

12. Tesio L. How specific is a medical speciality? A semiserious game to test your understanding of physical and rehabilitation medicine. Int $\mathrm{J}$ Rehabil Res 2012;35:378-81.

13. Jablonka E, Lamb MJ. Evolution in four Dimensions. Genetic, epigenetic, Behavioural and Symbolic Variation in the History of Life. Revised Edition. Cambridge, MA: MIT Press; 2014. 
14. Diamond J. Guns, Germs and Steel: The Fates of Human Societies. New York: W.W. Norton and Company; 1997.

15. Grmek MD. Disease at the dawn of the Western civilisation-in French; Payot Editions. Lausanne-Switzerland; 1983.

16. Canguilhem G. The Normal and the Pathological. Boston, MA, MIT Press; 1989 (original edition in French, 1966).

17. Agazzi E. Scientific Objectivity and its Contexts. Berlin, DE: Springer; 2014.

18. Berrios GE. Melancholia and depression during the $19^{\text {th }}$ century: A conceptual history. Br J Psychiatry 1988;153:298-304.

19. Buzzoni M. Mechanisms, experiments, and theory-ladenness: A realist-perspectivalist view. Axiomathes 2016;26:411-27.

20. Tesio L. Quality of life measurement: One size fits all. Rehabilitation medicine makes no exception. J Med Pers 2009;7:5-9.

21. Borsboom D, Mellenbergh GJ, van Heerden J. The theoretical status of latent variables. Psychol Rev 2003;110:203-19.

22. Bernard C. An Introduction to the Study of Experimental Medicine. Mineola, NY: Dover Books on Biology; 1957.

23. Cohen J. The earth is round $(P<.05)$. Am Psychol 1994;49:997-1003.

24. Grimby G, Tennant A, Tesio L. The use of raw scores from ordinal scales: Time to end malpractice? J Rehabil Med 2012;44:97-8.

25. Franchignoni F, Salaffi F, Tesio L. How should we use the visual analogue scale (VAS) in rehabilitation outcomes? I: How much of what? The seductive VAS numbers are not true measures. J Rehabil Med 2012;44:798-9.

26. Tesio L. Measuring person's behaviours and perceptions: Rasch analysis as a tool for rehabilitation research. J Rehabil Med 2003;35:1-11.

27. Tesio L. Outcome measurement in behavioural sciences: A view on how to shift attention from means to individuals and why. Int J Rehabil Res 2012;35:1-2

28. Tesio L, Scarano S. Individualized Coaching After Stroke Does Not Work How Much or Which One? Am J Phys Med Rehabil 2019. [In press].

29. Rothwell PM. Factors that can affect the external validity of randomised controlled trials. PLoS Clin Trials 2006;1:e9.

30. Smith GC, Pell JP. Parachute use to prevent death and major trauma related to gravitational challenge: Systematic review of randomised controlled trials. BMJ 2003;327:1459-61.

31. Shadish WR, Cook TD, Campbell DT. Experimental and Quasi-Experimental Designs for Generalized Causal Inference. Boston, MA: Houghton Mifflin Co.; 2002.

32. Mirabella G, Lebedev MA. Interfacing to the brain's motor decisions. J Neurophysiol 2017;117:1305-19.

33. Owen AM. Detecting consciousness: A unique role for neuroimaging. Annu Rev Psychol 2013;64:109-33.

34. Burkeman O. Why can't the world's greatest minds solve the mystery of consciousness? The Guardian; 21 January, 2015. Available from: https:// www.theguardian.com/science/2015/jan/21/-sp-why-cant-worldsgreatest-minds-solve-mystery-consciousness. [Last accessed on 2019 Mar 23].

35. Tesio L. Alternative medicines: Yes; alternatives to medicine: No. Am J Phys Med Rehabil 2013;92:542-5.

36. Levin JS, Glass TA, Kushi LH, Schuck JR, Steele L, Jonas WB, et al. Quantitative methods in research on complementary and alternative medicine. A methodological manifesto. NIH office of alternative medicine. Med Care 1997;35:1079-94.

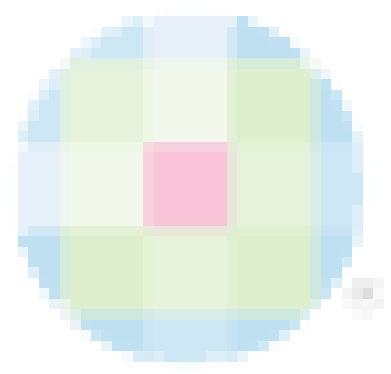




\section{Appendix 1 -Definition of PRM: A holistic or Simply a generic Picture?}

Considerable efforts are being spent internationally to define the scope of Physical and Rehabilitation Medicine (also said Physiatry) and the specific competences of the corresponding medical specialist, the Physiatrist. Subtle, yet relevant, differences lie in the sequence of words defining the specialty. The official European naming is "Physical and Rehabilitation Medicine" (P\&RM), while in US the official naming is Physical Medicine and Rehabilitation (PM\&R). For this reason, in the present article the neutral acronym PRM is adopted. The former naming, in the Author view, is preferable, because it implicitly highlights the intimate connection between physical means and rehabilitative goals of the discipline. Leaving aside this distinction, and neglecting whether the discipline in general or the corresponding medical specialist is dealt with, here below follow six examples showing definitions (in italics) of PRM. It is left to the reader to evaluate the consistency across definitions, and the specificity they assign to PRM compared to other medical specialties.

All websites have been last accessed on March 23 ${ }^{\text {rd }}, 2019$.

1) According to the Merriam-Webster dictionary, Physical Medicine and Rehabilitation is

a medical specialty concerned with preventing, diagnosing, and treating disabling diseases, disorders, and injuries by physical means (as by the use of electrotherapy, therapeutic exercise, or pharmaceutical pain control) - called also physiatry, physical medicine

(After: https://www.merriam-webster.com/dictionary/ physical\%20medicine \%20and\%20rehabilitation)

2) For the U.S. National Library of Medicine:

Physical medicine and rehabilitation, also referred to as physiatry or rehabilitation medicine, is a branch of medicine concerned with evaluation and treatment of, and coordination of care for, persons with musculoskeletal injuries, pain syndromes, and/or other physical or cognitive impairments or disabilities. The primary focus is on maximal restoration of physical and psychological function, and on alleviation of pain (adapted from definitions by the American Board of Medical Specialties and the American Board of Physical Medicine \& Rehabilitation).

(After: https://www.nlm.nih.gov/tsd/acquisitions/cdm/ subjects81.html)

3) The following statement, indireclty defining PRM, is produced by the leading international PRM society:

The mission of the International Society for Physical and Rehabilitation Medicine (ISPRM) is to optimize functioning and health-related quality of life and minimize disability in persons with disabilities and medical problems throughout the world (After: http://www.isprm.org/discover/mission-goals/)
4) This is the WHO definition of PRM:

Rehabilitation is a set of interventions designed to optimize functioning and reduce disability in individuals with health conditions in interaction with their environment. Health condition refers to disease (acute or chronic), disorder, injury or trauma. A health condition may also include other circumstances such as pregnancy, ageing, stress, congenital anomaly, or genetic predisposition. Rehabilitation thus maximizes people's ability to live, work and learn to their best potential. Evidence also suggests that rehabilitation can reduce the functional difficulties associated with ageing and improve quality of life

(After: https://apps.who.int/iris/bitstream/hand le/10665/254506/9789241549974-eng.pdf? sequence $=8$ )

5) Here is the definition of PRM in the European Union:

PRM is the primary medical specialty responsible for education and training [of] patients and health care providers, health promotion, prevention, medical diagnosis, functional assessment, treatment and rehabilitation management of persons of all ages experiencing disabling health conditions and their co-morbidities. After: European Physical and Rehabilitation Medicine Bodies Alliance. White Book on Physical and Rehabilitation Medicine in Europe. Introductions, Executive Summary, and Methodology. Eur J Phys Rehabil Med 2018;54:125-55. This White Book is freely available at: https://www.euro-prm.org/index.php?option=com content\&view=article \&id=111:3rd-edition-of-whitebookon-prm-published \&catid=22\&Itemid=136\&lang=en

6) The last example comes from a national Scientific society, i.e. the Italian Society of Physical and Rehabilitation Medicine (SIMFER):

The physiatrist is the medical specialist in physical and rehabilitation medicine. He/she has particular expertise in the treatment of disability caused by various diseases and / or pain, and has specific competences in the neuromuscular, osteoarticular, cognitive-relational, biomechanical and psychological-ergonomic fields. Helshe has unique knowledge about cardiovascular, respiratory, uro-gynecological, metabolic and nutritional functional problems following disability conditions. He/she has competences allowing to assess and address problems related to limitations of autonomy and participation of the Person, with respect to his/her physical, family, work and social environment. The physiatrist aims, therefore, at the highest possible recovery of functions and abilities, through a holistic approach to the person. The physiatrist works in an interprofessional and interdisciplinary team. He/she coordinates the team in the endeavour to realize the rehabilitation project with the goal of achieving functional outcomes through the highest possible recovery of functions and abilities of the Person.

(Authors' translation from Italian. After: https://www.simfer. it/chi-e-il-fisiatra/) 